\title{
Etude Histopathologique et Neurochimique Suite a des Lesions Unilaterales du Locus Coeruleus Chez le Rat et de la Region Postlocus Chez le Chat (Part 2)
}

\author{
R. MARCHAND, P.CHAUVEL, M. FANTINO, et L. J. POIRIER
}

SUMMARY: Unilateral lesions involving the area immediately caudal to the locus coeruleus in the cat are associated with slight decreases of noradrenaline (NA) in both sides of the spinal cord without any important change of serotonin (5-HT) concentration in the spinal cord and of $N A$ and 5-HT concentrations in the cerebral cortex of both sides. In other cats with similar lesions involving the same area bilaterally (postlocus lesions) NA is markedly decreased and 5-HT slightly decreased in the hypothalamus and spinal cord but the concentrations of NA and 5-HT of the cerebral cortex, striatum and thalamus are not conspicuously modified by such postlocus lesions.

Unilateral lesions in the area of the loci coeruleus and subcoeruleus in the rat are associated with a marked decrease of noradrenaline $(N A)$ in the ipsilateral cerebral cortex without any important change of NA in the contralateral cortex and in the spinal cord of both sides. Under such conditions the concentrations of serotonin $(5-H T)$ are not significantly, modified in the cerebral cortex and spinal cord of both sides. NA and 5-HT concentrations of the cerebral cortex of both sides are unmodified by unilateral lesions of the cerebellar nuclei in the rat. In the latter group of animals slight increases of NA and 5-HT in the spinal cord are significant in comparison to the values obtained in the control animals but not from one side of the spinal cord to the other.

From the Laboratoires de Neurobiologie, Hôpital de l'Enfant-Jésus et Faculté de Médecine, Université Laval, Québec, Qué. Canada.

Reprint requests to Dr. Louis J. Poirier, Laboratoires de Neurobiologie, Pavillon Notre-Dame, 2075 ave de Vitré, Québec, Qué. GIJ 5B3.

Supported by grant MT-732 from the Medical Research Council of Canada.
These results further support the suggestion that the noradrenergic coeruleocortical pathway originating in the loci coeruleus and subcoeruleus ascends on the same side and ends in the ipsilateral cerebral cortex. In the light of the results of this investigation and of those reported in a companion paper (Marchand et al., 1979) $N A$ fibers reaching the hypothalamus and spinal cord most likely arise from neurons located caudally in respect to the locus coeruleus. Moreover NA fibers ending in the hypothalamus do not ascend in the dorsolateral part of the isthmic area and, therefore, have a different course than the $N A$ fibers reaching the cerebral cortex and thalamus.

RÉSUMÉ: Une lésion unilatérale intéressant la région immédiatement caudale au locus coeruleus (postlocus) chez le chat n'entraîne qu'une faible baisse de la noradrénaline (NA) des deux côtés de la moelle épinière sans modification de la $N A$ du cortex cérébral et de la sérotonine (5$H T)$ corticale et spinale. Des lésions bilatérales du même type (postlocus) s'accompagnent de baisses très significatives de la NA et de légères baisses de la 5$H T$ au niveau spinal et hypothalamique sans modification importante de la NA et de la 5-HT corticales, striatales et thalamiques. Une lésion unilatérale intéressant la région du locus coeruleus et subcoeruleus chez le rat se traduit par une baisse très significative de la NA au niveau du cortex cérébral ipsilatéral sans modification importante de la NA au niveau du cortex contralatéral et de la moelle épinière des deux côtés.

Ces résultats étayent davantage l'hypothèse selon laquelle la voie NA coeruléocorticale qui tire son origine au niveau de neurones sis dans la région du locus coeruleus chemine du même côté et se termine dans le cortex cérébral homolatéral. Par ailleurs les résultats de cette étude et ceux découlant d'une étude connexe (Marchand et coll., 1979) suggèrent que les fibres NA destinées à l'hypothalamus et à la moelle épinière sont issues de périkarya situés caudalement à la région $d u$ locus. De plus les fibres NA qui atteignent l'hypothalamus n'empruntent pas la région dorsolatérale de l'isthme du rhombencéphale et, en conséquence, ont un trajet différent des fibres $N A$ destinées au cortex cérébral et au thalamus.

\section{INTRODUCTION}

Cette étude réalisée chez le chat et le rat est complémentaire à une recherche antérieure (Marchand et coll., 1979) réalisée seulement chez le chat où nous avons étudié les modifications morphologiques et neurochimiques induites par des lésions uni- et bilatérales provoquées $\mathrm{au}$ niveau et en avant de la région du locus coeruleus. Nous avions alors constaté qu'une lésion unilatérale sise au niveau du locus ou en avant s'accompagne d'une baisse très significative et soutenue de la noradrénaline (NA) exclusivement au niveau du cortex cérébral ipsilatéral sans modification importante de cette amine et de la sérotonine (5-HT) au niveau d'autres structures télencéphaliques et diencéphaliques et de la moelle épinière (Marchand et coll., 1979). Dans la présente étude nous comparons l'effet de lésions unilatérales du locus coeruleus et des noyaux profonds du cervelet au regard des concentrations de NA et de 5-HT de la moelle épinière et du cortex cérébral chez le rat. De plus nous avons cherché à préciser l'origine et le trajet de fibres monoaminergiques ascendantes et descendantes chez le chat à l'aide de 
lésions intéressant uni- et bilatéralement la zone de tissu nerveux sise à l'extrémité caudale du locus coeruleus et du locus subcoeruleus. Certains des animaux utilisés dans cette étude de corrélation morphologique et neurochimique ont fait l'objet d'enregistrements électrophysiologiques au regard de modifications de l'activité motrice associées aux perturbations du sommeil paradoxal chez le chat et l'épilepsie "myoclonique" expérimentale chez le rat.

\section{MATERIEL ET METHODES}

Trente-neuf chats et cinquante-deux rats ont été utilisés dans cette recherche. Dix-huit chats et quatorze rats n'ont subi aucun traitement et ont servi d'animaux contrôles. Onze chats ont subi une lésion unilatérale de la région immédiatement caudale à celle du locus coeruleus (lésion dite postlocus) alors que dix autres chats ont subi la même lésion mais des deux côtés du tronc cérébral. Trente-huit rats ont subi une lésion unilatérale intéressant soit la région du locus coeruleus (19 rats) soit la région des noyaux profonds du cervelet (19 rats). Les lésions ont été provoquées à l'aide d'un courant à haute fréquence et selon les paramètres décrits dans un article connexe (Marchand et coll., 1979). Les coordonnées stéréotaxiques consignées dans le tableau qui suit ont été prédéterminées selon l'atlas de Snider et Niemer (1961) pour le chat et celui de Skinner (1971) pour le rat:

$$
\underset{\text { Horizontal }}{\text { Plan }} \underset{\text { Sagittal }}{\text { Plan }} \quad \begin{gathered}
\text { Plan } \\
\text { Vertical }
\end{gathered}
$$

CHAT:

Région

"postlocus" $\quad-4.3 \quad 2.3 \quad-4.2$

$-4.3 \quad 2.3 \quad-4.8$

RAT:

Région

"locus"

Région

cérébelleuse

$\begin{array}{ccc}-1.8 & 1.3 & -2.5 \\ -3 & 2.2 & -1 \\ -3 & 3.5 & -1\end{array}$

Après une période de survie de trois à trente semaines, les animaux ont été sacrifiés par décapitation et les cerveaux et la moelle épinière furent rapidement prélevés. La partie du tronc cérébral contenant la lésion a été conservée et fixée dans du formol neutre $(10 \%)$ pour préparation de coupes sériées en vue de l'étude microscopique après coloration selon la méthode de Klüver et Barrera (1953) modifiée.

Chez le chat le cortex frontal et occipital, le striatum et le thalamus des deux côtés du cerveau et des segments de la moelle épinière sectionnée longitudinalement et l'hypothalamus indivis furent prélevés sur glace sèche et congelés en vue de la détermination chimique des monoamines. Chez le rat le cortex cérébral de chaque côté et des segments de la moelle épinière aussi sectionnée longitudinalement ont été prélevés et rapidement congelés pour détermination chimique subséquente des monoamines. Le dosage de la noradrénaline (NA) et de la sérotonine (5-HT) a été fait selon la méthode de Maickell et coll. (1968) et celui de la dopamine (DA) selon la méthode de Welch et Welch (1969). Toutes les données neurochimiques ont été analysées statisquement (student " $\mathrm{t}$ " test).

TABLE 1

Effects of brainstem unilateral lesions on the concentrations of noradrenaline (NA) and serotinin (5-HT) in the cerebral cortex and spinal cord of cats and rats.

\begin{tabular}{|c|c|c|c|c|}
\hline & \multicolumn{2}{|c|}{ Cerebral Cortex } & \multicolumn{2}{|c|}{ Spinal Cord } \\
\hline & NA & 5-HT & NA & 5-HT \\
\hline & UNL & UNL & UNL & UNL \\
\hline $\begin{array}{l}\text { CATS } \\
\text { Controls }\end{array}$ & $.57 \underset{(18)}{ \pm .04}$ & $.50 \underset{(18)}{ \pm .02}$ & $.32 \underset{(16)}{ \pm .02}$ & $\underset{(15)}{.85 \pm .05}$ \\
\hline Postlocus Region & $.57 \pm .08 \underset{(11)}{.59 \pm .06}$ & $.45 \pm .02$ (11) $.46 \pm .03$ & $.22 \pm .04 \quad .23 \pm .03$ & $.78 \pm .06 \pm \underset{(11)}{ } .86 \pm .08$ \\
\hline $\begin{array}{l}\text { RATS } \\
\text { Controls }\end{array}$ & $.70 \pm .06$ & $.73 \pm .03$ & $.52 \pm .04$ & $.73 \underset{(8)}{ \pm} .06$ \\
\hline $\begin{array}{l}\text { Locus Coeruleus } \\
\text { Region }\end{array}$ & $\underbrace{.23 \pm .02}_{* * * *} \quad .72 \pm .02$ & ${ }_{\dagger \dagger}^{.57 \pm .04} \underset{(19)}{.61 \pm .03}$ & $.49 \pm .04 \underset{(6)}{.60 \pm .04}$ & $.83_{(6)}^{.03} .81 \pm .08$ \\
\hline \multirow[t]{2}{*}{ Cerebellar Nuclei } & \multirow[t]{2}{*}{$.71 \pm .05 \underset{(19)}{.67 \pm .05}$} & \multirow[t]{2}{*}{$.64 \pm .03{ }_{\left({ }^{19}\right)}^{.67 \pm .04}$} & ${ }_{(10)}^{.69 \pm .03} .76 \pm .08$ & $.95 \pm .05_{(10)} .84 \pm .06$ \\
\hline & & & $\dagger \dagger$ & $\dagger \dagger$ \\
\hline
\end{tabular}

( ) number of animals; LES, UNL, lesioned and unlesioned side, resp.; \pm standard error ${ }^{*} \mathrm{p}<.05,{ }^{* * * *} \mathrm{p}<.0005$, LES against UNL side

$\dagger \mathrm{p}<.05, \uparrow \uparrow \mathrm{p}<.005,+\dagger \dagger \mathrm{p}<.0005$, LES or UNL against controls 


\section{RESULTATS}

Avant de décrire les changements histopathologiques et d'analyser les modifications au regard des structures nerveuses impliquées, il nous semble utile de faire ressortir certaines variations de concentrations régionales de monoamines mises en évidence au niveau de diverses structures du système nerveux central chez le chat et le rat.

D'une part la concentration moyenne de noradrénaline (NA) est plus élevée dans le cortex cérébral que dans la moelle épinière tant chez le chat $(.57 / .32 \mu \mathrm{g} / \mathrm{g})$ que chez le rat $(.70 / .52 \mu \mathrm{g} / \mathrm{g})$. D'autre part la concentration moyenne de sérotonine (5-HT) qui est moins élevée dans le cortex cérébral que dans la moelle épinière chez le chat $(.50 / .85 \mu \mathrm{g} / \mathrm{g})$ est égale dans les structures correspondantes chez le rat $(.73 / .73 \mu \mathrm{g} / \mathrm{g})$ (Tableau 1). Par ailleurs les concentrations moyennes de ces deux amines sont plus élevées dans le striatum et surtout dans l'hypothalamus du chat. La 5-HT est aussi plus élevée dans le thalamus que dans le cortex cérébral et la moelle épinière chez le chat. En effet les concentrations de la NA sont de $.60,1.09$ et $2.89 \mu \mathrm{g} / \mathrm{g}$ et celles de la 5 -HT de $1.26,1.32$ et $1.98 \mu \mathrm{g} / \mathrm{g}$ pour chacune de ces trois structures, respectivement (Tableau 2).

L'étendue des lésions et les structures en cause sont illustrées sur les figures 1-16.
A) LESIONS DE LA REGION IMMEDIATEMENT CAUDALE AU LOCUS COERULEUS OU LESIONS DITES "POSTLOCUS" CHEZ LE CHAT Aspect morphologique

Ce type de lésion avait pour but principal d'interrompre le plus extensivement possible les efférences descendantes des neurones du locus coeruleus et du locus subcoeruleus. Pour ce faire nous avons créé des lésions plus ou moins extensives intéressant la zone de tissu immédiatement caudale au locus coeruleus au niveau de la partie dorsolatérale de la protubérance. De telles lésions ont été provoquées unilatéralement chez 11 chats et bilatéralement chez 10 autres chats.

L'étude microscopique des cerveaux de chats porteurs de lésions dites "postlocus" montre que de telles lésions sont plus ou moins extensives d'un animal à l'autre. En particulier ces lésions détruisent une majeure partie de la région dorsolatérale du tegmentum du pons entre le niveau du noyau moteur (masticateur) du trijumeau (V) et la limite bulboprotubérantielle (Fig. 1-8). Ces lésions intéressent de façon plus ou moins importante le tegmentum au niveau de la moitié caudale du pons. Elles interrompent les différents groupes de fibres qui y cheminent et en particulier les divers contingents de fibres ascendantes et descendantes du faisceau tegmentaire central et elles détruisent certains groupes de neurones notamment ceux du noyau pontis caudalis. Chez quelques animaux de ces deux groupes de chats porteurs de lésions uni- ou bilatérales la partie tout à fait caudale du locus coeruleus et du locus subcoeuruleus est envahie par le processus lésionnel. Par contre certaines de ces lésions qui toutes détruisent une zone de tissu nerveux caudale au locus et commune à tous les animaux, s'étendent soit plus médialement vers la région du raphé correspondant, soit plus ventralement où elles envahissent une partie plus importante du tegmentum pontique (Fig. 1-8) ou encore plus caudalement jusqu'au niveau du genou du nerf facial sur le plancher du IVe ventricule (Fig. 8). Par contre ces lésions épargnent les voies cérébellofuges y compris celles du pédoncule cérébelleux supérieur (voir aussi les légendes accompagnant les figures 1-8).

\section{Corrélations morphologiques et neurochimiques}

Une lésion unilatérale détruisant de façon plus ou moins extensive la zone de tissu nerveux caudale au locus coeruleus (lésion dite "postlocus") (Fig. 1-4) n'entraîne aucune modification significative des concentrations de noradrénaline (NA) et de sérontonine (5-HT) du cortex cérébral des deux côtés du cerveau. Cependant ces lésions dont certaines sont relativement extensives s'accompagnent d'une

TABLE 2

Effects of bilateral post-locus lesions on the concentrations $(\mu \mathrm{g} / \mathrm{g})$ of noradrenaline (NA) and serotonin (5-HT) in the cerebral cortex, striatum, thalamus, hypothalamus and spinal cord of cats.

\begin{tabular}{|c|c|c|c|c|c|c|}
\hline & & Cortex & Striatum & Thalamus & Hypothalamus & Spinal Cord \\
\hline \multirow[t]{2}{*}{ NA } & CONTROLS & $.57 \underset{(18)}{ \pm .04}$ & $\underset{(18)}{1.09 \pm .11}$ & $.60 \pm .06$ & $\underset{\left({ }^{18}\right)}{2.89 \pm .22}$ & $.32 \pm .22$ \\
\hline & LESIONS & $\begin{array}{c}.45 \underset{(10)}{ \pm} .04 \\
\dagger\end{array}$ & $\begin{array}{c}1.09 \pm .20 \\
(10)\end{array}$ & $.51 \underset{\left({ }^{10}\right)}{ \pm} .04$ & $\begin{array}{c}1.56 \pm .21 \\
(10) \\
\dagger \dagger \dagger\end{array}$ & $\begin{array}{c}.14 \pm .02 \\
\left({ }^{9}\right) \\
\dagger \dagger \dagger\end{array}$ \\
\hline \multirow[t]{2}{*}{ 5-HT } & CONTROLS & $.50 \underset{(18)}{ \pm .02}$ & $\underset{(18)}{1.32 \pm .07}$ & $1.26 \pm .06$ & $1.98 \pm .13$ & $.85 \pm . .05$ \\
\hline & LESIONS & $\begin{array}{c}.50 \pm .05 \\
\left({ }^{10}\right)\end{array}$ & $\begin{array}{c}1.29 \pm .17 \\
\left({ }^{10}\right)\end{array}$ & $\begin{array}{c}1.45 \pm .12 \\
\left({ }^{10}\right)\end{array}$ & $\begin{array}{c}1.65 \pm .12 \\
\left({ }^{10}\right) \\
\dagger\end{array}$ & $\begin{array}{c}.67 \pm .03 \\
(10) \\
\dagger \dagger\end{array}$ \\
\hline
\end{tabular}

( ), number of animals; \pm standard error

( ), number of animals; \pm standard error

$\dagger \mathrm{p}<.05$, $\dagger \dagger \mathrm{p}<.005$, $\dagger \dagger \dagger \dagger \mathrm{p}<.0005$ 

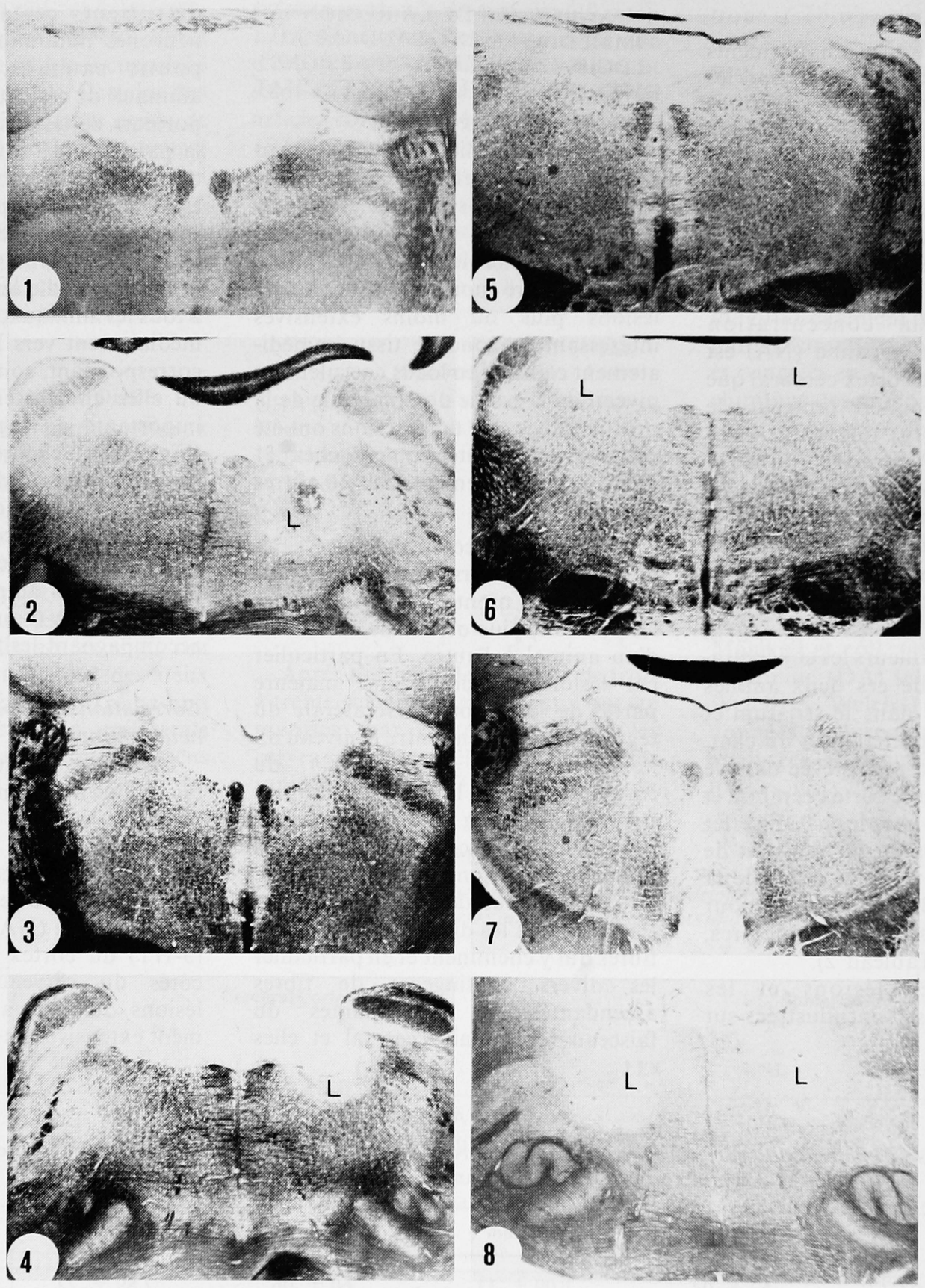

PLATE 1

Explanation of Figures

Microphotographs of transverse sections through pons of the brains of cats illustrating unilateral and bilateral lesions of the "postlocus" type. Cresyl violet and fast blue. Magnif. x 8.5

1-4: Unilateral lesions caudal to the locus coeruleus in cats $\# 384(1,2)$ and $\# 739$ $(3,4)$. The larger lesion in cat $\# 384$ extends more ventrally $(2)$. This type of lesion more especially involves the central tegmental tract and a more or less important part of the corresponding pontine tegmentum.

5-8: Bilateral lesions of the "postlocus" type in cats $\# 618(5,6)$ and $\# 960(7,8)$. The larger lesions in cat $\# 960$ destroy a more important area of the caudal part of the pontine tegmentum (8). 
baisse faiblement significative $(p<.05)$ de la NA de la moelle épinière des deux côtés sans modification significative de la 5-HT au niveau de la même structure chez ce groupe d'animaux ayant survécu 2 à 30 semaines après la lésion (Tableau 1).

Des lésions semblables mais intéressant les deux côtés du tronc cérébral à la fois (Fig. 5-8) entraînent une très légère baisse mais significative $(p<.05)$ de la NA corticale sans modifications importantes des concentrations moyennes de la NA striatale et thalamique du cerveau. De plus des lésions bilatérales "postlocus" s'accompagnent d'une diminution très significative $(p<.0005)$ des concentrations de NA de l'hypothalamus et de la moelle épinière (Tableau 2). Ces modifications ne sont pas fonction du temps de survie (2 à 14 semaines) d'un animal à l'autre. Par ailleurs les mêmes lésions bilatérales (postlocus) ne modifient pas significativement la sérontonine (5-HT) du cortex, du striatum et du thalamus alors que chez les mêmes animaux l'hypothalamus et surtout la moelle épinière accusent une baisse significative $(\mathrm{p}<.05$ et $\mathrm{p}<.005$, respectivement) de la 5-HT (Tableau 2).

La légère baisse de NA corticale constatée à la suite de ces lésions est plus évidente $(\mathrm{p}<.005)$ au niveau du cortex occipital (.38 \pm .03 comparativement à $.51 \pm .03 \mu \mathrm{g} / \mathrm{g}$ chez les contrôles) qu'au niveau du cortex frontal $(.51 \pm .07$ comparativement à $.63 \pm .04$ chez les contrôles). Dans les mêmes conditions (lésions postlocus bilatérales) les concentrations de 5-HT du cortex frontal et du cortex occipital ainsi que les concentrations de dopamine (DA) striatale n'accusent aucun changement significatif par rapport aux animaux contrôles (résultats non consignés dans les tableaux).

\section{B) LESIONS DE LA REGION DU} LOCUS COERULEUS CHEZ LE RAT

\section{Aspect morphologique}

Chez 19 rats la lésion unilatérale, de façon générale plus extensive que les lésions correspondantes provoquées chez le chat, intéresse la partie dorsolatérale de l'isthme du rhombencéphale et son extension caudale au niveau de la partie rostrale du pons (Fig. 9-14). Chez tous les animaux la majorité des neurones du locus coeruleus et du locus subcoeruleus sont détruits. Seule la partie caudale du locus coeruleus est épargnée chez certains individus; cependant les axones ascendants de ces neurones, selon toute évidence, sont interrompus rostralement où la lésion est relativement extensive. Chez la plupart de ces animaux la lésion détruit de façon extensive le pédoncule cérébelleux supérieur et ses noyaux associés (parabrachialis medialis et lateralis), le noyau et la racine mésencéphalique du trijumeau, le noyau latérodorsal de l'isthme, ainsi que le faisceau tegmentaire central du côte correspondant. De plus la lésion intéresse la partie latérale de la substance grise périventriculaire, la partie tout-à-fait dorsolatérale du tegmentum pontique notamment au niveau du noyau moteur (masticateur) du $\mathrm{V}$ et occasionnellement la zone susjacente du cervelet (Fig. 9-14) (voir aussi les légendes des figures 9-14).

\section{Corrélations morphologique et neurochimique}

Chez les 19 rats de ce groupe la lésion unilatérale qui en particulier intéresse les neurones du locus coeruleus et du locus subcoeruleus entraîne une baisse (de l'ordre de 65\%) trè significative $(\mathrm{p}<.0005$ par rapport aux valeurs contrôles et à celles du côté non lésé) de la noradrénaline (NA) du cortex cérébral contralatéral à la lésion (Tableau 1). Par ailleurs elle s'accompagne de légères baisses de la NA spinale ipsilatéralement à la lésion et de la 5-HT des deux côtés du cortex. Cependant la légère baisse de la NA spinale du côté lésé n'est pas significative par rapport aux témoins. Par contre la moëlle épinière des deux côtés n'accuse aucune modification significative de la 5-HT (Tableau 1) suite à de telles lésions chez le rat. De plus l'importance de la baisse de la NA corticale du côté lésé n'est pas fonction du temps de survie ( 2 à 7 semaines). La présence d'un foyer cortical au cobalt chez certains animaux (lésés et non lésés) n'affecte pas la concentration de NA, si cette zone est exclue du prélèvement, au regard des valeurs obtenues chez les autres animaux.

\section{C) LESIONS CEREBELLEUSES CHEZ LE RAT}

Des lésions cérébelleuses unilatérales ont été provoquées chez 19 rats après avoir constaté que les lésions dorsolatérales de la région du locus coeruleus détruisaient de façon plus ou moins extensive le pédoncule cérébelleux supérieur chez la majorité des animaux. Ces lésions unilatérales intéressent de façon plus ou moins extensive les noyaux profonds du cervelet ainsi que le tissu avoisinant (Fig. 15, 16) (voir aussi les légendes de ces figures).

\section{Corrélations morphologiques et neurochimique}

De telles lésions unilatérales réalisées chez 19 rats n'entraînent après 2 à 6 semaines de survie aucune baisse de concentrations de noradrénaline et de sérotonine tant au niveau du cortex cérébral qu'au niveau de la moëlle épinière des deux côtés. Cependant de faibles augmentations de la NA et de la 5-HT spinales ont été constatées après ces lésions (Tableau 1). De même la présence d'un foyer au cobalt exclus du prélèvement du tissu cortical destiné aux déterminations chimiques chez quelques rats n'affecte pas les concentrations moyennes de ces amines à la lumière des valeurs obtenues chez les autres animaux (témoins et lésés).

\section{DISCUSSION}

Les résultats d'une étude connexe réalisée chez le chat (Marchand et coll., 1979) et ceux obtenus chez le rat dans la présente étude nous ont permis de constater que la baisse de la noradrénaline (NA) corticale associée à une lésion unilatérale de la région du locus coeruleus met en cause le cortex cérébral ipsilatéral et n'affecte pas la concentration de NA du cortex contralatéral chez ces deux espèces. Ces résultats rejoignent ceux d'autres auteurs (Arbuthnottet coll., 1973; Korf et coll., 1973a, b; Kobayashi et coll., 1974; Ross et Reiss, 1974; Worth et coll., 1976; Jones et col., 1977) à la suite de travaux impliquant divers métabolites de la NA chez le rat. Il en découle, sur la foi de nombreuses données neurochimiques, que la voie dite "coeruleo-corticale" (Maeda et Shimizu, 1972; Maeda et coll., 1973) 


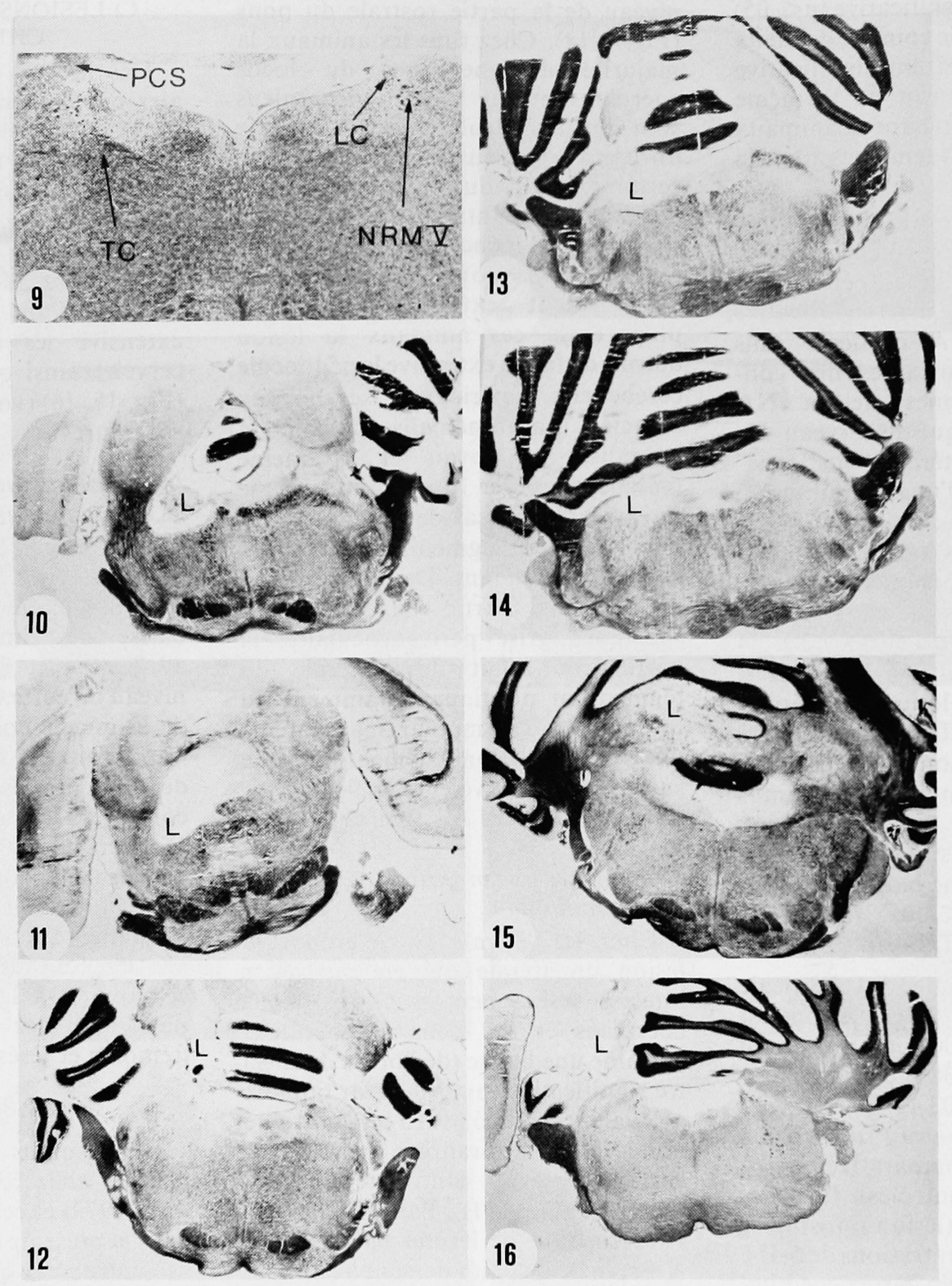

PLATE 2

Explanation of Figures

Microphotographs of transverse sections through the cerebellum and brain stem of rats illustrating unilateral lesions in the area of the locus coeruleus and of the cerebellar nuclei. Cresyl violet and fast blue. Magnif. x 18 (9) and x 8.5 (10-16).

9

Area of the loci coeruleus and subcoeruleus in a normal rat. LC, locus coeruleus; NRMV, nucleus of the mesencephalic root of V; PCS, superior cerebellar penduncle; TC, central tegmental tract.

10-14: Unilateral lesions in the area of the loci coeruleus and subcoeruleus in rats LC 2 (10), LC 3(11), LC 4 (12) and LD 3(13,14). The lesions involve in addition to the above mentioned structures the superior cerebellar peduncle, the central tegmental tract, the laterodorsal nucleus and a small zone of the pontine tegmentum. Moreover the lesion extends in the cerebellum on the corresponding side in rat $\mathrm{LC} 4(12)$.

15-16: Unilateral lesions in the area of the cerebellar nuclei in rats LC 6 (15) and B $2(16)$. 
dont les fibres contribuent au faisceau "noradrénergique dorsal" (Ungerstedt, 1971) est constituée en très grande majorité de fibres qui cheminent et se terminent ipsilatéralement à leur origine. Par contre, la distribution de ces fibres, de très petit diamètre (de l'ordre de $.35 \mu \mathrm{m}$ au niveau cortical selon Descarries et coll. 1977) a été étudiée par les méthodes d'histofluorescence, d'autoradiographie ou encore à l'aide de la peroxydase du raifort (HRP) ou de la technique de mise en évidence des fibres en dégénérescence par imprégnation à l'argent (Fink et Heimer 1967). Les données découlant de cette dernière approche et visant à identifier les fibres noradrénergiques ascendantes après lésions de neurones correspondants, telle que réalisée par Shimizu et coll. (1974) et McBride et Sutin (1976), sont mises en doute par plusieurs chercheurs dont Gatter et Powell (1977) et Hedreen (1978). En effet le faible calibre des fibres monoaminergiques en général nous porte à croire que ces fibres NA risquent de ne pas être mises en évidence par les techniques faisant appel à l'imprégnation argentaffine et, en conséquence, il est plus que probable que les fibres en dégénérescence révélées par cette technique, à la suite de lésions au niveau du locus coeruleus ou de la substance noire par exemple, impliquent des fibres de passage de plus gros calibre qui, selon toute évidence, ne peuvent pas être entièrement épargnées en créant des lésions au niveau de telles structures. Par ailleurs une majorité des travaux faisant appel à l'approche autoradiographique et mettant en cause soit des acides aminés (leucine- ${ }^{3} \mathrm{H}$, proline $-{ }^{3} \mathrm{H}$ ) ou des précurseurs marqués de la NA ( $L$ DOPA $-{ }^{3} \mathrm{H}$, dopamine- ${ }^{3} \mathrm{H}$ ) tendent à démontrer que les fibres noradrénergiques issues de la région du locus coeruleus cheminent et se terminent ipsilatéralement à leur origine (Segal et coll. 1973; Pickel et coll. 1974; Levin et Stolk 1977). Cependant Jones et coll. (1977) et Jones et Moore (1977) faisant appel à la proline- ${ }^{3} \mathrm{H}$ la leucine${ }^{3} \mathrm{H}$ et la $\mathrm{L}-D O P A-{ }^{3} \mathrm{H}$ et mesurant la radioactivité en aval du site d'injection (Jones et coll. 1977) ou étudiant le trajet des fibres marquées (Jones et Moore 1977) rapportent que le locus coeruleus projette abondamment aux structures ipsilatérales du tronc cérébral, du diencéphale et du télencéphale et aussi, mais à un degré moindre aux structures contralatérales de ces mêmes régions. Au contraire, sur la base de mesures biochimiques, ces mêmes auteurs ont cependant constaté une baisse exclusivement ipsilatérale de la NA corticale suite à une lésion de la région $\mathrm{du}$ locus coeruleus correspondant (Jones et coll. 1977). De plus, les documents microphotographiques et autres produits par ces auteurs, qui semblent reposer surtout sur l'emploi de la proline- ${ }^{3} \mathrm{H}$ et à un degré moindre de la L-DOPA- ${ }^{3} \mathrm{H}$ sont en opposition avec ceux de Segal et coll. (1973) et Pickel et coll. (1974) qui utilisant aussi la proline- ${ }^{3} \mathrm{H}$ chez la même espèce constatent que le locus coeruleus projette exclusivement dans les structures ipsılatérales du télencéphale et du diencéphale. Jones et coll. (1977) et Jones et Moore (1977) qui citent dans leurs travaux ces derniers auteurs ne trouvent pas moyen dans leurs deux longs articles de mentionner et discuter ces résultats tout-à-fait contradictoires. En général les résultats découlant de l'application de la méthode d'histofluorescence telle que développée par Hillarp et ses collaboratuers (Falck et coll. 1962) tendent à démontrer que la voie noradrénergique coeruleo-corticale qui projette de façon diffuse sur toutes les régions du cortex cérébral (Anden et coll. 1966; Ungerstedt 1971; Maeda et Shimizu 1972) est constituée de fibres ascendantes à majorité si non en exclusivité ipsilatérales (Ungerstedt 1971; Maeda et Shimizu 1972; Maeda et coll. 1973). Par ailleurs les études impliquant l'utilisation de la peroxydase du raifort ou "horseradish peroxidase (HRP)" ne simplifient pas le problème de l'existence ou la nonexistence de fibres coeruleo-corticales ascendant contralatéralement à leurs origines. A la suite d'injections de HRP au niveau du cortex cérébral du singe Freedman et coll. (1975) constatent qu'après injection unilatérale de HRP au niveau de diverses régions du cortex cérébral chez le singe écureuil les neurones du locus coeruleus contralatéral sont marqués (rétrogradement) dans une proportion de $50 \%$ de ceux du locus coeruleus ipsilatéral à l'injection. De même Kievit et Kuypers (1975) étudiant le cortex moteur et Gatter et Powell (1977) étudiant diverses régions du cortex cérébral chez le macaque ont aussi constaté le marquage de neurones du locus coeruleus contralatéral en plus de ceux du locus coeruleus ipsilatéral suite à l'injection intracorticale de HRP. Cependant Gatter et Powell (1977) qui ont étudié de façon beaucoup plus systématique les diverses régions du cortex cérébral ont rapporté que la voie coeruleocorticale est surtout constituée de fibres cheminant ipsilatéralement à leur origine bien qu'ils aient remarqué que dans la majorité des cas environ $20 \%$ des neurones du locus coeruleus contralatéral au cortex injecté réagissaient positivement au HRP. Par ailleurs Llamas et coll. (1975) utilisant la "HRP" chez le chat concluent à l'existence de projections coeruleo-corticales principalement ipsilatérales. De plus seul le cortex frontal et plus particulièrement le "gyrus proreus" correspondant au cortex préfrontal recevrait des fibres directes en provenance de la région du locus coeruleus. D'autre part McBride et Sutin (1976) constatent après injection de HRP au niveau de la partie médiane (noyaux intralaminaires et hypothalamiques et région préoptique latérale) du diencéphale chez le chat, que le marquage n'affecte presque exclusivement que les neurones des locus coeruleus et subcoeruleus et du noyau parabrachial latéral ipsilatéraux. Ils en concluent à l'existence de fibres coeruleodiencéphaliques prennant la précaution, cependant, de signaler que la HRP peut être captée par les fibres de passage lésées sur la foi des travaux de DeVito et coll. (1974). En étudiant les afférences du cortex visuel (aires 17, 18) chez le chat par la technique de la peroxydase du raifort, Léger et collaborateurs (1975) ont mis en évidence une projection du locus coeruleus en très grande partie homolatérale au site d'injection. Pour expliquer la présence d'un apport d'origine contralatérale à l'innervation $\mathrm{du}$ cortex visuel, résultats qu'ils considèrent en contradiction avec ceux obtenus par les techniques 
d'histofluorescence, ces auteurs évoquent la présence possible de neurones non-catécholaminergiques au niveau de la région du locus coeruleus projetant bilatéralement au cortex visuel.

De l'ensemble de ces observations relativement contradictoires surtout en ce qui a trait à l'existence de fibres coeruleo-corticales destinées au cortex cérébral de l'hémisphère opposé il nous apparait prématuré de concluse présentement à leur existence (du mons en nombre important) ou non. Cependant d'un point de vue fonctionnel, il appert que la voie coeruleo-corticale, impliquent divers groupes de neurones noradrénergiques de la région du locus coeruleus, est surtout ipsilatérale comme en témoignent les données neurochimiques suite à des lésions uni- et bilatérales chez diverses espèces. Cette dernière éventualité en ce qui a trait à l'existence d'une voie coeruleocorticale NA directe et ipsilatérale est davantage étayée par les résultats obtenus suite à des lésions intéressant d'un ou des deux côtés la région dite praelocus chez le chat (Marchand et coll., 1979). En effet de telles lésions qui épargnent les neurones NA euxmêmes entraînent une baisse non moins significative de la NA corticale que celles détruisant de façon extensive la région du locus coeruleus. Les résultats obtenus chez ces animaux (Marchand et coll., 1979) étayent ceux de Jones et coll. (1973), et de Blondeaux et coll. (1975) qui ont constaté chez le chat une baisse significative de la NA corticale après lésions bilatérales intéressant soit le tegmentum mésencéphalique ou l'isthme du rhombencéphale. En revanche l'absence de modification importante de la NA corticale suite à une lésion unilatérale intéressant la région "postlocus" chez le chat ou les noyaux du cervelet chez le, rat ou encore suite à des lésions bilatérales de la région "postlocus" chez le chat, telle que rapportée dans cette étude, renforce l'hypothèse selon laquelle les neurones NA qui contribuent à la voie coeruleo-corticale naissent exclusivement de la région latérodorsale de la protubérance rostrale correspondant plus précisement à l'isthme du rhombencéphale.
De plus il nous faut préciser que les neurones du locus coeruleus projettent à des structures du voisinage tant au niveau du mésencéphale, de la protubérance et du bulbe qu'au niveau du cortex cérébelleux comme en font foi les travaux de Hillarp et coll. (1966) Anden et coll. (1967), Loizou (1969) Olson et Fuxe (1971) et Ungerstedt (1971) chez le rat et ceux de Maeda et coll. (1973) et Chu et Bloom (1974) chez le chat. Dans le même ordre d'idée nous avons constaté au cours de cette étude que contrairement à des lésions de type locus et praelocus (Marchand et coll., 1979), des lésions bilatérales et relativement extensives intéressant la zone de tissu nerveux sise en arrière du locus coeruleus s'accompagnent d'une baisse très significative de la concentration de la noradrénaline hypothalamique sans pour autant entraîner de modifications très importantes de cette amine au niveau du thalamus, du striatum et du cortex cérébral. Ces résultats qui sont en accord avec la présence d'une voie noradrénergique destinée à l'hypothalamus et prenant naissance au niveau bulbopontique latéral (Olson et Fuxe 1972; Maeda et coll., 1973; Poitras, 1977) et, donc indépendante de la voie coeruleocorticale, soulignent l'organisation topographique particulière de divers groupes neuronaux monoaminergiques chez le chat (Poitras et Parent 1978) contribuant à diverses structures cibles par l'intermédiare de voies ascendantes et descendantes relativement distinctes. De même $\mathrm{Chu}$ et Bloom (1974) faisant appel à l'histofluorenscence ont constaté que les neurones CA sis en arrière du locus coeruleus dans le pons caudal ne sont pas affectés par des lésions relativement extensives du mésencéphale dorsal qui cependant perturbent l'activité des autres groupes neuronaux CA de la région du locus chez le chat. Les résultats obtenus chez le chat porteur de lésions de la région du locus (Marchand et coll., 1979) et ceux obtenus chez le rat dans cette étude tendent à démontrer que l'apport du locus coeruleus à l'élaboration de NA spinale est nul ou à tout le moins proportionnellement peu important. En effet, les concentrations de NA spinale observés après lésions unilatérales du locus coeruleus chez le chat ne sont pas modifiées significativement alors qu'après lésions bilatérales du locus l'on observe une légère baisse de cette amine au niveau spinal (Marchand et coll., 1978). Ces résultats supportent davantage le point de vue de Carlsson et coll. (1964), Dahlstron et Fuxe (1965) et Ungerstedt (1971) qui décrivent une voie noradrénergique bulbo-spinale. Si tel est le cas et en accord avec les travaux de Satoh et coll. (1977) qui ont fait appel au HRP en association avec la mise en évidence de l'activité MAO au niveau du locus, les neurones marqués par le HRP dans la région du locus coeruleus suite à l'injectin de cette substance au niveau de la moëlle épinière chez le chat (Kuypers et Maisky 1975) risquent de ne pas être de nature noradrénergique. En effet nous n'avons constaté une baisse très significative de la NA spinale qu'apprés des lésions bilatérales intéressant la moitié caudale de la partie dorsolatérale du pons (lésions dites postlocus). Par ailleurs nos résultats soulignent qu'à l'encontre de la voie coeruleo-corticale qui est exclusivement ipsilatérale, la voie ponto et bulbo-spinale noradrénergique a une distribution bilatérale.

Nous avons signalé dans une étude antérieure (Marchand et coll., 1979) et dans la présente étude que l'analyse détaillée des modifications neurochimiques associées à diverses lésions intéressant la partie dorsolatérale au niveau ponto-mésencéphalique, nous révéle que les changement neurochimiques observés au niveau de diverses structures cibles sont, à toute fin pratique, de même ordre après deux ou trois semaines qu'après trente semaines ou plus. En conséquence les modifications neurochimiques engendrées par de telles lésions semblent irréversibles et permanentes tout comme il en est de la dopamine (DA) striatale suite à la destruction du mécanisme nigrostrié (Poirier et Sourkes 1965; Poirier et coll. 1966, 1969). Aussi les récupérations fonctionnelles que l'on peut observerà la suite de processus lésionnels impliquant ces mécanismes ne peuvent pas être imputées à la restoration d'activités métaboliques mettant en cause ces mécanismes monoaminer- 
giques. Cependant à notre avis il ne faut pas exclure la possibilité que, lors de la production brutale de lésions, comme c'est le cas dans la majorité des lésions provoquées expérimentalement, divers groupes de fibres et de neurones y compris les structures monoaminergiques au voisinage de telles lésions puissent être transitoirement impliqués et, donc peuvent du même coup inactiver, mais de façon réversible certaines activités métaboliques.

\section{REFERENCES}

ANDEN, N. -E., DAHLSTRÖM, A., FUXE, K.. LARSSON, K., OLSON, L. and UNGERSTEDT, U. (1966). Ascending monoamine neurons to the telencephalon and diencephalon. Acta Physiol. Scand., 67: 313-326.

ANDEN, N. -E.. FUXE. K. AND UNGERSTEDT, U. (1967). Monoamine pathways to the cerebellum and cerebral cortex. Experientia, 23: 838-839.

ARBUTHNOTT, G. W., CHRISTIE, J. E., CROW, T. J. ECCLESTON, D. and WALTERS, D. S. (1973). Lesions of the locus coeruleus and noradrenaline metabolism in cerebral cortex. Exp. Neurol., 41: 4I1-417.

BLONDAUX, C., BUDA, M., PETITJEAN, F. et PUJOL, J. F. (1975). Hypersomnie par lésion isthmique chez le chat. I. Etude du métabolisme des monoamines cérébrales. Brain Res., 88: 425-437.

CARLSSON, A., FALCK, B., FUXE, K. and HILlARP, N. -A. (1964). Cellular localization of monoamines in the spinal cord. Acta physiol. scand., 60: 112-119.

CHU, N. -S. and BLOOM, F. E. (1974). The catecholamine-containing neurons in the cat dorsolateral pontine tegmentum: distribution of the cell bodies and some axonal projections. Brain Research; 66: 221.

DAHLSTROM, A. and FUXE, K. (1965). Evidence for the existence of monamine neurons in the central nervous system. II. Experimentally induced changes in the intraneuronal amine levels of bulbospinal neuron systems. Acta physiol scand., 64, Suppl. 247: 1-36.

DESCARRIES, L., WATKINS, K. C. and LAPIERRE, Y. (1977). Noradrenergic axon terminals in the cerebral cortex of rat. III. Topometric ultrastructural analysis. Brain Research, 133: 197-222.

DeVITO, J. I., CLAUSING, K. W. and SMITH, O. A. (1974). Uptake and transport of horseradish peroxidase by cut end of the vagus nerve. Brain Res., 82: 269-271.
FALCK, B., HILlARP, N. A., THIEME, G. and TORP, A. (1962). Fluorescence of catecholamines and related compounds condensed with formaldehyde. J. Histoch. Cytochem., 10: 348-354.

FINK, R. P. and HEIMER, L. (1967). Two methods for selective silver impregnation of degenerating axons and their synaptic endings in the central nervous system. Brain Res., 4: 369-374.

FREEDMAN, R., FOOTE, S. L. and BLOOM F. E. (1975). Histochemical characterization of a neocortical projection of the nucleus locus coeruleus in the squirrel monkey. $J$. comp. Neur., 164: 209-232.

GATTER, K. C. and POWELL, T. P.S. (1977). The projection of the locus coeruleus upon the neocortex in the macaque monkey. Neuroscience, 2: 441-445.

HILLARP, N. -A., FUXE, K. and DAHLSTROM, A. (1966). Demonstration and maping of central neurons containing dopamine, noradrenaline and 5-hydroxytryptamine and their reactions to psychopharmaca. Pharm. Rev., 18: 727-741.

HEDREEN, J. (1978). Brainstem projections of rat substantia nigra. Anat. Rec., 190: 417.

JONES, B. E., BOBILLIER, P., PIN, C. and JOUVET, M. (1973). The effects of lesion of catecholamine containing neurons upon monoamine content of the brain and EEG and behavioural waking in the cat. Brain Res., 58: 157-177.

JONES, B. E., HALARIS, A. E., McILHANY, M. and MOORE, R. Y. (1977). Ascending projections of the locus coeruleus in the rat. I. Axonal transport in central noradrenaline neurons. Brain Res., 127: 1-21.

JONES, B. E. and MOORE, R. Y. (1977). Ascending projections of the locus coeruleus in the rat. II. Autoradiographic study. Brain Res., 127: 23-53.

KIEVIT, J. and KUYPERS, H. G. J. M. (1975). Basal forebrain and hypothalamic connections to frontal and parietal cortex in the rhesus monkey. Science, 187: 660-662.

KLUVER, H. and BARRERA, E. (1953). A method for the combined staining of cells and fibers in the nervous system. J. Neuropath. exp. Neurol., 12: 400-403.

KOBAYASHI, R. M., PALKOVITS, M., KOPIN, I. and JACOBIWITZ, D. (1974). Biochemical mapping of noradrenergic nerves arising from the rat coeruleus. Brain Res., 77: 269-279.

KORF, J., AGHAJANIAN, G. K. and ROTH, R. H. (1973a). Stimulation and destruction of the locus coeruleus: opposite effects on 3methoxy 4-hydroxyphenylglycol sulfate levels in the rat cerebral cortex. Europ. J. Pharmacol., 21: 305-310.

KORF, J., ROTH, R. H. and AGHAJANIAN, G. K. (1973b). Alterations in turnover and endogenous leves of norepinerphrine in cerebral cortex following electrical stimulation and acute axotomy of cerebral noradrenergic pathways. Europ. J. Pharmacol., 23: 276-282.
KUYPERS, H. G. J. M. and MAISKY, V. A. (1975). Retrograde axonal transport of horseradish peroxidase from spinal cord to brain stem cell groups in the cat. Neurosci. Lett., 1: 9-14.

LEGER, L., SAKAI, K., TOURET, M., SALVERT, D. et JOUVET, M. (1975). Mise en évidence par la technique de peroxydase de projections directes entre le tegmentum ponto-mésencéphalique et le cortex visuel chez le chat. C.R. Acad. Sci. (Paris), 281: 1159-1162.

LEVIN, B. E. and STOLK, J. A. (1977). Axoplasmic transport of norepinephrine in the locus coeruleus-hypothalamic system in the rat. Brain Res., 120: 303-315.

LLAMAS, A., REINOSO-SUAREZ, F. and MARTINEZ-MORENO, E. (1975). Projections to the gyrus proreus from the brain stem tegmentum (locus coeruleus, raphe nuclei) in the cat, demonstrated by retrograde transport of horseradish peroxidase. Brain Res., 89: 331-336.

LOIZOU, L. A. (1969). Projection of the nucleus locus coeruleus in the albino rat. Brain Res., 15: 563-566.

MAEDA, T., PIN, C., SALVERT, D., LIGIER, M. et JOUVET, M. (1973). Les neurones contenant des catécholamines du tegmentum pontique et leurs voies de projection chez le chat. Brain Res., 57: 119 152.

MAEDA, T. et SHIMIZU, N. (1972). Projections ascendantes du locus coeruleus et d'autres neurones aminergiques pontiques au niveau du prosencéphale du rat. Brain Res., 36: 19-35.

MAICKEL, R. P., COX, R. H. Jr., SAILLANT, J. and MILLER, F. P. (1968). A method for the determination of serotonin and norepinephrine in discrete areas of rat brain. Int. J. Neuropharmacol., 7: 275-281.

MARCHAND, R., FANTINO, M., DANKOVA, J. et POIRIER, L. J. (1979). Corrélations histopathologiques et neurochimiques en fonction de lésions de la région du locus coeruleus chez le chat. Can. J. Neur. Sc. 6: $27-38$

McBRIDE, R. L. and SUTIN, J. (1976). Projections of the locus coeruleus and adjacent pontine tegmentum in the cat. J. Comp. Neur., 165: 265-284.

OLSON, L. and FUXE, K. (1971). On the projection from the locus coeruleus noradrenaline neurons: the cerebellar innervation. Brain Res., 28: 165-171.

OLSON, L. and FUXE, K. (1972). Further mapping out of central noradrenaline neuron systems: projections of the "subcoeruleus" area. Brain Res., 43: 389395

PICKEL, V. M., SEGAL, M. and BLOOM, F. E. (1974). A radioautographic study of the efferent pathways of the nucleus coeruleus. J. comp. Neur., 155: 15-42. 
POIRIER, L. J., BOUVIER, G., BEDARD, P., BOUCHER. - R., LAROCHELLE, L., OLIVIER, A. and SINGH, P. (1969). Essai sur les circuits neuronaux impliqués dans le tremblement postural et l'hypokinésie. Rev. Neurol., 120: 15-40.

POIRIER, L. J. and SOURKES, T. L. (1965). Influence of the substantia nigra on the catecholamine content of the striatum. Brain, 88: 181-192.

POIRIER, L. J., SOURKES, T. L., BOUVIER, G., BOUCHER, R. and CARABIN, S. (1966). Striatal amines, experimental tremor and the effect of harmaline in the monkey. Brain, 89: 37-52.

POITRAS, D. (1977). Etude histochimique et bioçhimique de la distribution et des projections des neurones monoaminergiques du tronc cérébral et de l'hypothalamus chez le chat. Thèse, Québec, 199pp.
POITRAS, D. and PARENT, A. (1978). Atlas of the distribution of monoamine-containing nerve cell bodies in the brain stem of the cat. J. comp. Neur., 179: 699-718.

ROSS, R. A. and REIS, D. J. (1974). Effects of lesions of locus coeruleus on regional distribution of dopamine- $\beta$-hydroxylase activity in rat brain. Brain Res., 73: 161-166.

SATOH, K., TOHYAMA. M., YAMAMOTO, K., SAKUMOTO, T., SHIMIZU, N. (1977). Noradrenaline innervation of the spinal cord studied by the horse-radish peroxidase method combined with monoamine oxidase staining. Exp. Brain Res., 30: 175-186.

SEGAL, M., PICKEL, V. and BLOOM, F. E. (1973). The projections of the nucleus locus coeruleus: an antoradiographic study. Life Sci., 13: 817-821.

SHIMIZU, N., OHNISHI, S., TOHYAME, M. and MAEDA, T. (1974). Demonstration by degeneration silver method of the ascending projection from the locus coeruleus. Exp. Brain Res., 20: 181-192.
SKINNER, J. E. (1971). Neuroscience: $A$ Laboratory Manual. Philadelphia, Saunders.

SNIDER, R. S. and NIEMER, W. T. (196I) A stereotaxic atlas of the cat brain. The University of Chicago Press, Chicago.

UNGERSTEDT, U. (1971). Stereotaxic mapping of the monoamine pathways in the rat brain. Acta physiol. scand., Suppl. 367: 1-48.

WELCH, A. S and WELCH, B. L. (1969). Solvent extraction method for simultaneous determination of norepinephrine, dopamine, serotonin and 5-hydroxyindoleacetic acid in a single mouse brain. Anal. Biochem., 30: $161-179$.

WORTH, W. S., COLLINS, J., KETT, D. and AUSTIN, J. H. (1976). Serial changes in norepinephrine and dopamine in rat brain after locus coeruleus lesions. Brain Res., 106: 198-203. 